\title{
CRYSTALLOGRAPHY OF ALUMINA-YAG EUTECTIC
}

\author{
S.C. Farmer and Ali Sayir \\ NASA Glenn Research Center \\ 21000 Brookpark Rd. \\ Cleveland, $\mathrm{OH} 44135$
}

Robert M. Dickerson

Lawrence E. Matson

Materials Science and Technology Div.

WL/MLLM

Los Alamos National Laboratory

Los Alamos, NM 87545

Wright-Patterson AFB

Dayton, $\mathrm{OH}$

\begin{abstract}
Multiple descriptions of the alumina-YAG eutectic crystallography appear in the ceramic literature. The orientation between two phases in a eutectic system has direct impact on residual stress, morphology, microstructural stability, and high temperature mechanical properties. A study to demonstrate that the different crystallographic relationships can be correlated with different growth constraints was undertaken. Fibers produced by Laser-Heated Float Zone (LHFZ) and Edge-defined Film-fed Growth (EFG) were examined. A map of the orientation relationship between $\mathrm{Al}_{2} \mathrm{O}_{3}$ and $\mathrm{Y}_{3} \mathrm{Al}_{5} \mathrm{O}_{12}$ and their relationship to the fiber growth axis as a function of pull rate are presented. Regions in which a single orientation predominates are identified.
\end{abstract}

\section{INTRODUCTION}

The structure and properties of $\mathrm{Al}_{2} \mathrm{O}_{3}-\mathrm{Y}_{3} \mathrm{Al}_{5} \mathrm{O}_{12}$ eutectic materials have received considerable attention as potentially strong, creep resistant structural materials. In studies of mechanical properties of directionally solidified eutectic fibers, fracture origins and unusual distortion effects correspond with abrupt changes in the eutectic lamellae structure [1]. A detailed examination of the eutectic crystallography of these fibers using transmission electron microscopy revealed multiple orientation relationships between phases.

The orientation relationship (O.R.) between constituent phases of a eutectic system has direct impact on residual stress, morphology, microstructural stability, and high temperature mechanical properties. A number of different orientation relationships for the alumina-YAG system have already been published. High-resolution electron microscopy and interpretation of results using geometrical criteria by Hay and Matson [2] and by Mallamaci et al. [3] present three different orientation relationships and work by Sayir et al. [1] presents a fourth. More recently, examination of mechanically tested fibers at NASA Glenn corroborated the O.R. reported by Sayir et al., and by Mallamaci, but they were not the predominant configuration in these fibers.

The objective of the work reported here was to demonstrate the unusually strong dependence of the eutectic orientation relationship on growth conditions in the alumina-YAG eutectic system. A map of the orientation relationship between $\mathrm{Al}_{2} \mathrm{O}_{3}$ and $\mathrm{Y}_{3} \mathrm{Al}_{5} \mathrm{O}_{12}$ and their relationship to the fiber growth axis as a function of pull rate will be presented and regions in which a single orientation predominates are identified.

This is a preprint or reprint of a paper intended for presentation at a conference. Because changes may be made before formal publication, this is made available with the understanding that it will not be cited or reproduced without the permission of the author. 


\section{EXPERIMENTAL PROCEDURE}

Eutectic fibers produced both by edge defined film fed growth and by laser-heated float zone (LHFZ) methods have been studied. At NASA Glenn, a series of LHFZ materials grown from a common feed rod $\left(\mathrm{Al}_{2} \mathrm{O}_{3}-18.7 \mathrm{~m} / \mathrm{o} \mathrm{Y}_{2} \mathrm{O}_{3}\right.$ composition) and differing only in pull rate were produced. Fibers were pulled at speeds of $3.8,7.6,38$ and $152 \mathrm{~cm} / \mathrm{hr}$. Fiber diameters ranged from 175 to $225 \mu \mathrm{m}$. Longitudinal and transverse sections were characterized by means of large angle tilting experiments in a conventional transmission electron microscope (Philips 400T, 120 $\mathrm{kV}$ ). Microdiffraction patterns were obtained from coincident zone axes. Standard stereographic methods were used for determining habit plane and approximate growth axis [4].

RESULTS

$$
\text { É? }
$$

Fibers examined at different pull rates exhibicathangef in approximate growth direction (the crystallographic direction most nearly parallel to the fiber axis), habit plane and in the orientation relationship between alumina and YAG. For ease of comparison, results are described in terms of directions parallel to the alumina basal plane normal [0001], close-packed oxygen direction [10$10]$ and the $a_{2}$-lattice direction [-12-10] which form an orthogonal set. Four-index Miller-Bravais notation to describe the trigonal alumina structural cell [5] will be used throughout.

Of the materials examined, the $3.8 \mathrm{~cm} / \mathrm{hr}$ and $152 \mathrm{~cm} / \mathrm{hr}$ LHFZ materials appear to be "single crystal" in nature, i.e., a single orientation relationship and a consistently observed crystallographic orientation with respect to the fiber growth axis. Characterization in these cases is straightforward. Material grown at $7.6 \mathrm{~cm} / \mathrm{hr}$ is very similar to that grown at $3.8 \mathrm{~cm} / \mathrm{hr}$ except that interruptions of the "single crystal" character are occasionally observed. The $38 \mathrm{~cm} / \mathrm{hr}$ (LHFZ) material possesses mixed character exhibiting more than one morphology and orientation relationship. The heterogeneous fibers are less suited to characterization by TEM due to the limited amount of material sampled by this technique. EFG fibers used in mechanical testing at Glenn had more uniform microstructures, but also exhibited multiple O.R.'s A larger number of EFG fibers were examined, and therefore results for these mixed character fibers are reported with greater confidence.

LHFZ fibers grown at the slowest rates, $3.8^{4}$ exhibit a single orientation relationship between phases, a well-defined $(0001):\{211\}$ habit plane between alumina and YAG and a consistent growth direction with respect to the fiber axis. The morphology of materials grown at $3.8 \mathrm{~cm} / \mathrm{hr}$, Figure 1a, consists of faceted alumina rods, $5-7 \mu \mathrm{m}$ wide, in a continuous YAG matrix. The orientation relationship is:

$$
\begin{aligned}
\text { O.R. (a) } & <0001>:<2-1-1> \\
& <10-10>:<111> \\
& <1-210>:<0-11>
\end{aligned}
$$

which corresponds to OR2 of Hay and Matson[2]. The approximate growth axis is $<10-10\rangle:\langle-111\rangle$ in agreement with Dickey et al. [6]. Neighboring rods are relatget by reflection about (0001) indicating that YAG is the leading phase. The c-axis of alumina is -perpendicular to the growth axis, a condition commonly observed in boules grown by Verneuil methods [7].

The crystallography and morphology of the fastest grown fiber, $152 \mathrm{~cm} / \mathrm{hr} p 4 h$ rate, differ greatly from those observed at slower rates of growth. The $\mathrm{c}$-axis has rotated from -perpendicular to the fiber growth axis to a -parallel position. The liquid solid interface has faceled as can be inferred from the eutectic morphology, Figure $1 \mathrm{~b}$. With the alumina trigonal axis aligned along the growth axis, three symmetry-related facets develop. The facet planes are presumably the rhombohedral (10-12) planes which have been identified as the liquid-solid interface facet planes in c-axis grown alumina EFG crystals [8,9]. The eutectic microstructure consists of alumina "trefoils" containing very fine, highly ordered arrays of YAG rods or lamellae of $-100 \mathrm{~nm}$ dimension, more than an order of magnitude finer scale than occurs in $3.8 \mathrm{~cm} / \mathrm{hr}$ material. The rod 
morphology is observed on all large facets. The YAG and alumina crystal structures are oriented such that maximum symmetry is preserved:

$$
\text { O.R. (b) } \begin{aligned}
<0001>: & <111> \\
& <10-10>:<01-1> \\
& <1-210>\text { : }<-211>
\end{aligned}
$$

YAG lamellae have a well-defined $(10-10):\{01-1\}$ habit plane. Only this single orientation relationship between alumina and YAG was observed.

This orientation relationship, O.R. (b), is described by Dickerson in reference [1] and is restated here in terms of its highest symmetry elements. In his work, fine lamellae were observed on the surface of a melt-grown fiber which badly distorted during creep. The region consisted of alumina lamellae in a continuous YAG matrix as indicated by neighboring alumina variants being crystallographically related by reflection about $(0001)$. Dickerson was the first to report the "apparent twin relationship" between neighboring alumina lamellae, Figure 2*.
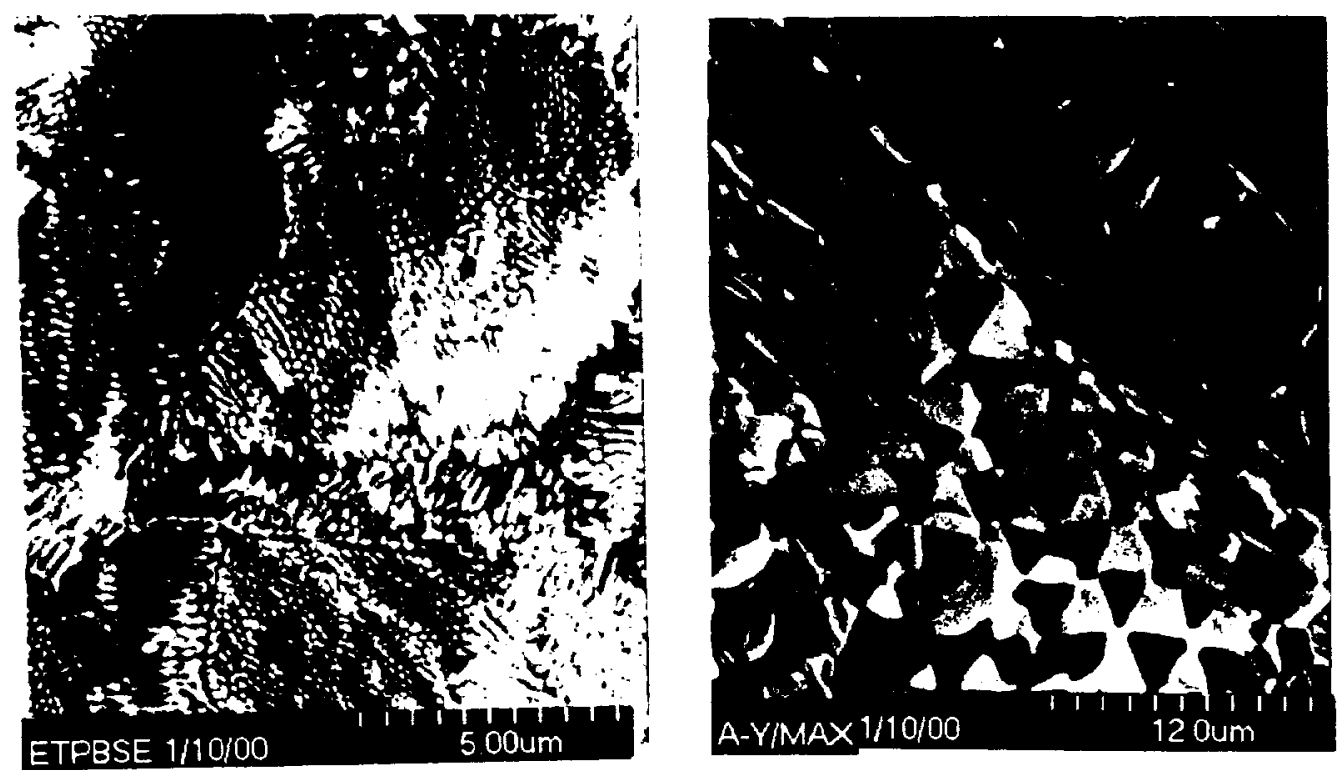

Fig. la) transverse section SEM of $3.8 \mathrm{~cm} / \mathrm{hr}$ LHFZ fiber showing faceted alumina rods (dark) in continuous YAG matrix (light). 1b) transverse section of $152 \mathrm{~cm} / \mathrm{hr}$ fiber. Interface facets produce three symmetry-related faces about the trigonal axis. YAG rods $\sim 10 \mathrm{~nm}$ diameter form on the facet faces.

The morphologies of fibers grown at intermediate growth rates $(38.1 \mathrm{~cm} / \mathrm{hr})$ are highly variable and multiple orientation relationships are expressed within a single fiber, Figure $3 a$ and $b$. The high symmetry O.R. (b) is observed but is not most prevalent. Regions of multiple variants of YAG in a continuous alumina matrix and alumina "twin-related" variants within a continuous YAG matrix both occur. The two conditions are readily distinguished in diffraction. The c-axis of alumina is inclined with respect to the growth axis. Two additional O.R. are observed. In one, the eutectic solidifies without a well-defined habit plane:

* [note: a longer paper. in progress. will present additional diffraction patterns and additional SEM photos] 


$$
\text { O.R. (c) }<\begin{aligned}
<0001>: & <312> \\
& <10-10>:<2-4-1> \\
& <1-210>:<11-2>
\end{aligned}
$$

The O.R. (c) was observed in both YAG matrix and alumina matrix configurations. A second O.R. is observed in regions with a well-defined $(0001):\{11-2\}$ habit plane and reflection-related alumina variants in a continuous YAG matrix. This O.R. is:

$$
\text { O.R. (d) } \begin{aligned}
<001> & :<11-2\rangle \\
& <10-10>:<312> \\
& <1-210>:<2-4-1>
\end{aligned}
$$

As extensive mechanical properties studies have been done on EFG fibers of mixed character, we include some comments here. Thermal gradients are typically an order of magnitude lower than obtained in LHFZ growth. Fibers, which were mechanically tested, had mixed textures. O.R. (c) which grows without a well-defined interface was predominant, but regions of (b) and (d) are also present. The microstrucure can be described as irregular lamellar. In some fiber sections, a large eutectic grain with a different O.R. and different $c$-axis orientation formed at or near the exterior surface and severe distortion during high temperature creep conditions results.
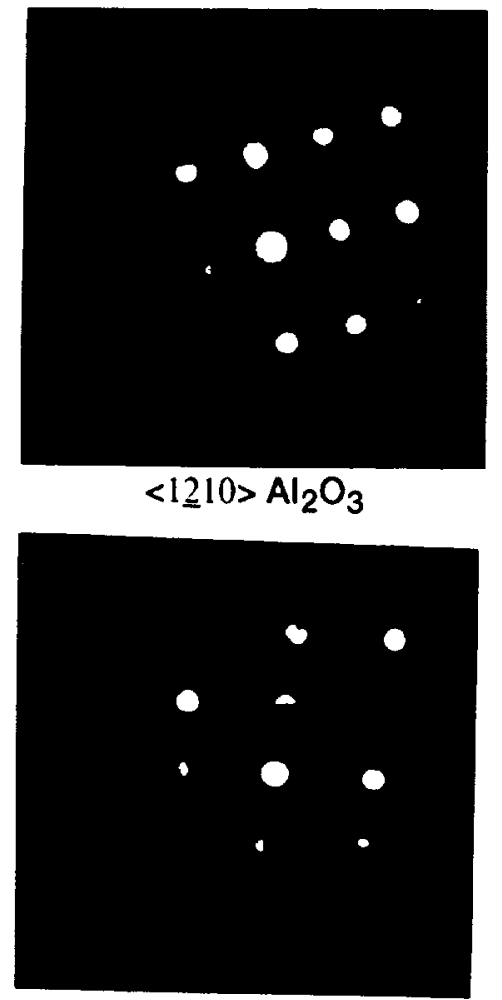

$<\underline{12} \underline{1} 0>\mathrm{Al}_{2} \mathrm{O}_{3}$

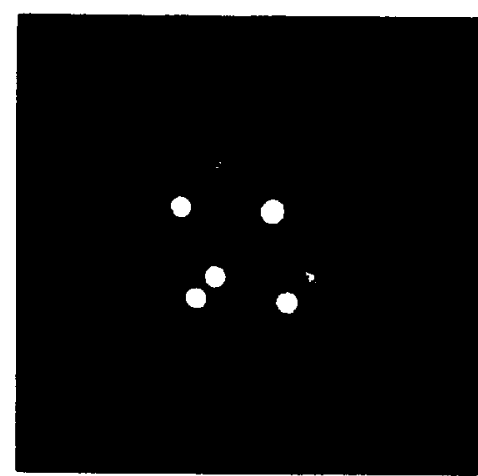

$<112>$ YAG

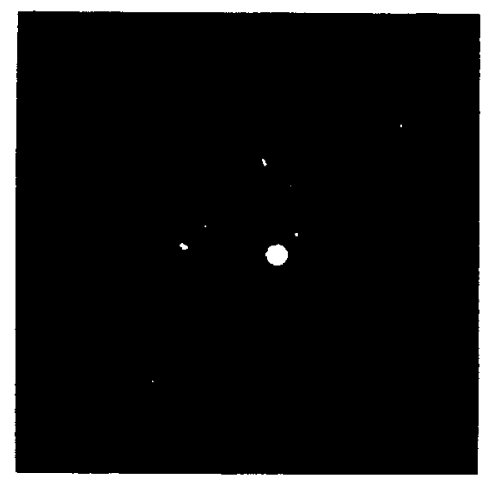

SAD (Superposition)

Fig. 2 Diffraction patterns from a region of O.R. (b) tilted to coincident zone axes. Patterns from alumina lamellate on either side about a common Y'AG lamellace are related by reflection about (0001). The common $(0001):\{11-1\}$ reciprocal vector is mathed with a white arrowhead.

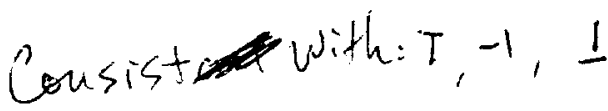



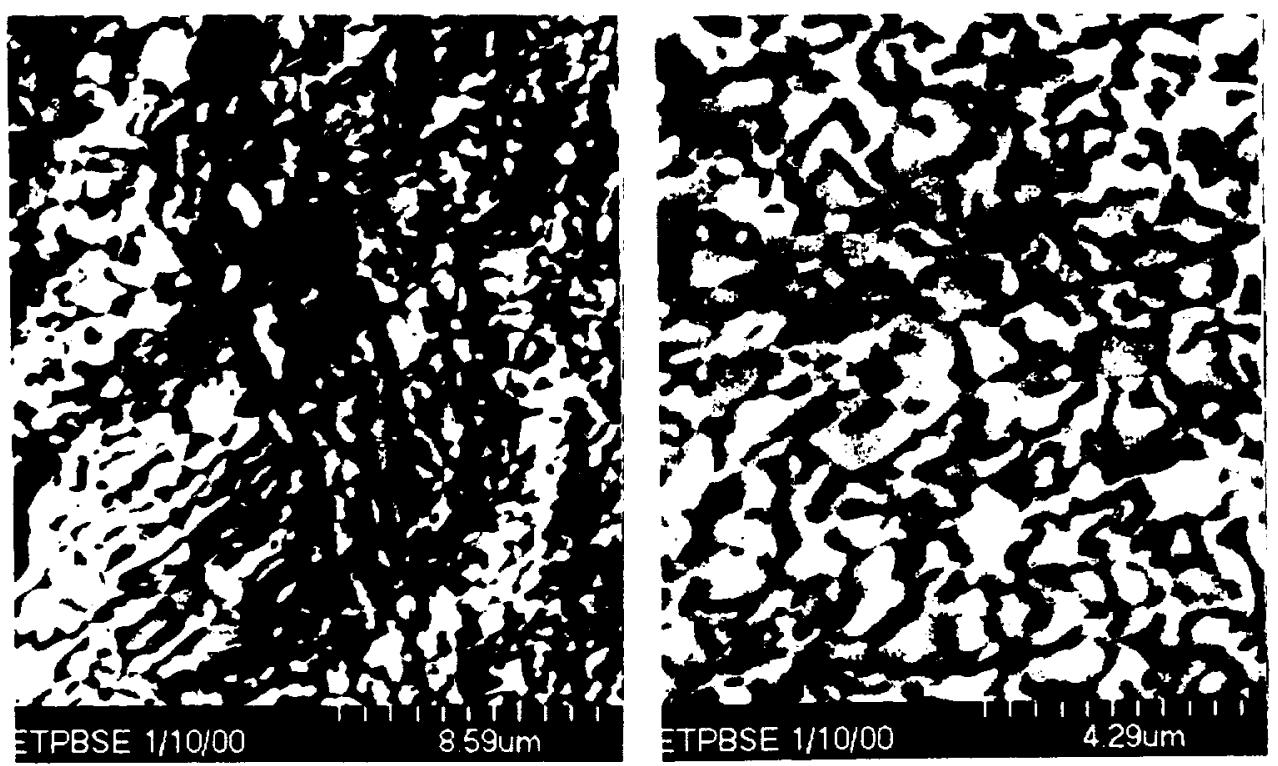

Fig. 3 Two SEM photos from different regions of fiber grown at intermediate rates, $38.1 \mathrm{~cm} / \mathrm{hr}$. Variable microstructure and multiple O.R.'s are characteristic of these fibers.

\section{INTERPRETATION OF RESULTS}

The above results can be interpreted in the context of general eutectic phenomenology where the growth kinetics, rather than interface energy minimization, is the determining factor in microstructural development $[10,11]$. During eutectic growth, the structure of the solidifying system evolves over time and the configuration that grows at the fastest velocity or with the greatest intrusion distance into the liquid becomes dominant [11]. (A low energy interface between constituent phases, but not necessarily the minimum energy interface, is a contributing factor.) A eutectic system can exhibit more than one characteristic microstructure as a function of the imposed heat and mass transfer constraints. The microstructure, which can grow at the lowest liquid-solid interface undercooling, is preferred [11]. The two phases of the eutectic are arranged with the sluggish phase in the most advantageous configuration at the solid liquid front [11]. In other words, the sluggish phase leads or grows as rods.

As stability of the solidification front is often discussed in terms of $G / V$ where $G$ is the temperature gradient in the liquid ahead of the growing solid and $\mathrm{V}$ the solidification rate. A map of morphology and crystallography of the alumina-YAG system could be presented in a fashion analogous to Chadwick [12], Figure 4, where morphology stability domains for a eutectic system are indicated for 1) degenerate or faceted 2) irregular lamellar, and 3)fibrous or faceted as a function of increasing growth velocity. Direct analogy between the description of these morphologic domains and the velocity dependence of the alumina-YAG eutectic at the 3.8 and $152 \mathrm{~cm} / \mathrm{hr}$ pull rates can be made.

At low pull rates, the crystal faces with lowest liquid-solid energies preferentially form. Characteristic faceled shapes and growth directions evolve which reflect the anisotropy of the liquid-solid interface. When a facet is also a low energy interface plane. formation of a welldetined habit between phases more readily occurs. A change in the prevalence of planar interfaces 
between alumina and YAG with an increase of pull-rate (decreased faceting) can be seen by comparing Figure 1a and Figure 3. The rubric that "growth occurs on close-packed planes in close-packed directions" obtains for alumina under slow growth conditions. Alumina grows in the close-packed [10-10] direction and the (0001) close-packed basal plane of alumina forms the interface between alumina and YAG. That YAG, the sluggish phase, leads is indicated by its effect on crystallography. The adjacent alumina rods are (0001) reflection-related variants in a continuous YAG phase.

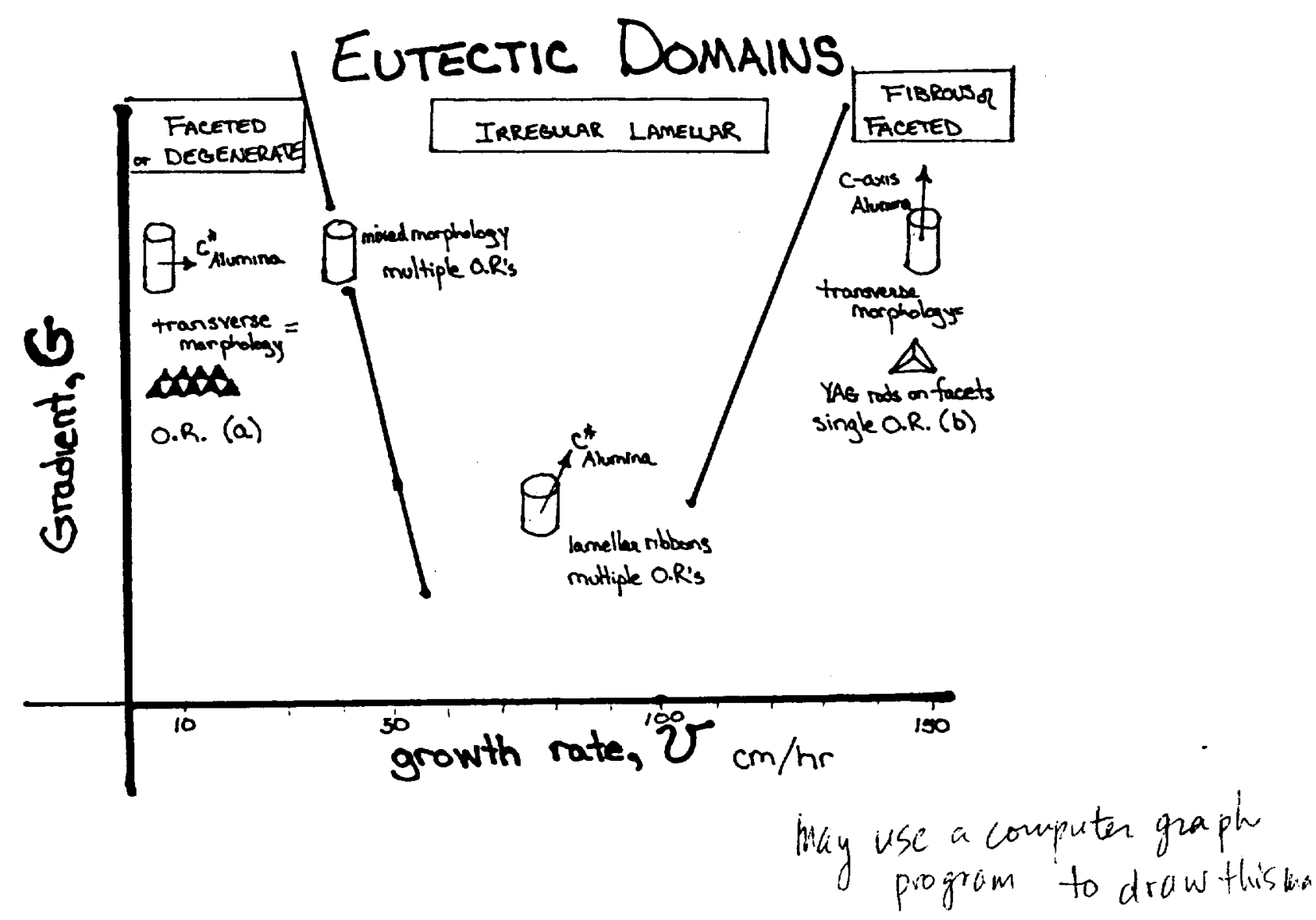

Fig. 4 Microstructural results for this initial study projected onto a Eutectic Domain Map.

At the highest pull rate, the anisotropy of energy of attachment dominates. The energy to nucleate a step on a flat surface is higher than the energy to propagate a step by adding atoms at a ledge. Large facets form, Figure ib. The trefoil or trigonal cell morphology indicates that the continuous alumina phase is determining the eutectic morphology and thus also must be the leading phase. Both rod and lamellar YAG are present with rods on the larger facets. The faceted portion of the liquid-solid interface lags unfaceted portions making diflusion of excess solute away from the growing facet surface more difficult. For geometrical reasons. lateral diffusion of 
excess solute away from rods is more efficiently accomplished than two-dimensional lateral diffusion from lamellae and therefore rods preferentially occur on all large facets.

Interpreting the results in the intermediate growth regimes is less clear. A region of regular lamellar growth was not observed in LHFZ fibers. The $38.1 \mathrm{~cm} / \mathrm{hr}$ fibers exhibit regions of degenerate and of lamellar morphology and appear to be at or near a growth rate at which transition to a lamellar morphology may occur. Additional research on materials grown at pull rates between 38.1 and $152 \mathrm{~cm} / \mathrm{hr}$ is required.

A pictorial summary of results is shown in Figure 4 and represents an initial attempt at establishing a Eutectic Domain map for the alumina-YAG eutectic system. This may serve as a guide in understanding the crystallographic texture of these materials. which is necessary for accurate interpretation of mechanical data and for modeling of mechanical behavior.

\section{CONCLUSIONS}

The crystallographic texture of alumina-YAG eutectic fibers is highly dependent on processing conditions. That alumina-YAG eutectic fibers undergo a change in morphology, crystallography and growth axis as a function of pull rate has been demonstrated. Single crystallographic relationships can be obtained at both low and high pull rates. The orientation of the alumina caxis with respect to the fiber growth axis in these two cases differs by $90^{\circ}$. Due to the strong anisotropy of creep behavior for alumina, this is expected to have important consequences on the high temperature deformation behavior of these materials. Intermediate pull rates produce additional orientation relationships between the alumina and YAG phases and mixed textures. Fibers with mixed textures can experience severe distortion at high temperatures.

References:

'A. Sayir, R.M. Dickerson, H.M. Yun, S. Heidger and L.E. Matson, "High Temperature Mechanical Properties of Directionally solidified $\mathrm{Al}_{2} \mathrm{O}_{3} / \mathrm{Y}_{3} \mathrm{Al}_{5} \mathrm{O}_{12}(\mathrm{YAG})$ Eutectic fibers", 7th Annual HITEMP Review, 74-1 to 74-12 (1994).

${ }^{2}$ R.S. Hay and L.E. Matson, "Alumina/Yttrium-Aluminum Garnet Crystallographic Orientation Relationships and Interphase Boundaries: Observations and Interpretation by Geometric Criteria", 39 [8] 1981-1994 (1991).

'M.P. Mallamaci, J. Bentley and C.B. Carter, "In-situ TEM Crystallization of Silicate-Glass Films on $\mathrm{Al}_{2} \mathrm{O}_{3}$ ", Acta mater. 46 [1] 283-303 (1998).

${ }^{4} \mathrm{~J}$. W. Edington, "Monographs in Practical Electron Microscopy in Materials Science, N.B. Philips, Eindhoven (1975).

"M.L. Kronberg, "Plastic Deformation of Single Crystals of Sapphire: Basal Slip and Twinning", Acta Metall. 5 507-524 (1957).

"E.C. Dickey, C.S. Frazer, T.R. Watkins, and C.R. Hubbard, "Residual Stresses in Hightemperature Ceramic Eutectics", J. Eur. Cer. Soc., 19 2503-2509, (1999).

"Synthetic Gems Production Techniques", Chemical Technology Review No. 149. ed. L.H. Yaverbaum, Noyes Data Corporation. New Jersey (1980).

${ }^{*}$ V.A. Tatarchenko. T.N. Yalovets. G.A. Satunkin, L.M. Zatulovsku. L.P. Egorov and D.Ya. Kravetsky. "Defects in Shaped Sapphire Crystals". J. Crystal Growth 50 335-340 (1980).

"J.T.A. Pollock. "Filamentary Sapphire, Part 1". J. Mat.Sci.7 631-648 (1972).

I"R. Elliott. "Eutectic Solidification Processing", Butterworths \& Co Ltd., London (1983).

"W.A. Tiller, "The Science of Crystallization, Macroscopic Phenomena and defect Generation", Cambridge University Press. Cambridge (1991). 
${ }^{12}$ G.A. Chadwick, "Progress in materials science", 12; Ed. B. Chalmers, Pergammon Press, London, 1963. 